\title{
PATOGENICIDADE DE NEMATÓIDES ENTOMOPATOGÊNICOS A COCHONILHA-DA- RAIZ-DO-CAFEEIRO DYSMICOCCUS TEXENSIS (TINSLEY) (HEMIPTERA: PSEUDOCOCCIDAE) EM LABORATÓRIO
}

\author{
V.S. Alves ${ }^{1}$, A. Moino Junior ${ }^{1}$, L.V.C. Santa-Cecilia² ${ }^{2}$ V. Andaló ${ }^{1}$, G.C. Souza ${ }^{1}$
}

${ }^{1}$ Universidade Federal de Lavras, Departamento de Entomologia, CP 37, CEP 37200-000, Lavras, MG, Brasil. Email: vivialves21@hotmail.com

RESUMO

A cochonilha-da-raiz-do-cafeeiro Dysmicoccus texensis (Tinsley) ataca as raízes desta planta, podendo causar sérios danos às plantações e conseqüentes perdas na produção. Os nematóides entomopatogênicos (NEPs) são agentes eficientes no controle de várias pragas encontradas no solo, o que sugere provável eficiência no controle da cochonilha-da-raiz-do-cafeeiro. Dessa forma, este trabalho teve por objetivo avaliar a patogenicidade de alguns isolados de NEPs à cochonilhada-raíz-do-cafeeiro através de testes de seleção de isolados, determinação da concentração letal máxima $\left(\mathrm{CLM}_{99}\right)$ e patogenicidade a criptas coletadas no campo. Os isolados Heterorhabditis sp. CCA, Heterorhabditis bacteriophora, Heterorhabditissp. JPM3.1 eHeterorhabditis sp. JPM3 foram os que apresentaram maior virulência, alcançando valores máximos de mortalidade de 100, 94, 94 e 82\%, respectivamente, na maior concentração testada (100 juvenis infectantes (JI)/inseto). A CL ${ }_{99}$ determinada para Heterorhabditis sp. CCA foi estimada em $530 \mathrm{JI} /$ placa. Valor semelhante foi encontrado para Heterorhabditis sp. JPM3 que teve a $\mathrm{CL}_{99}$ igual a $560 \mathrm{JI} /$ placa. Estimando a concentração por área, o valor obtido para Heterorhabditis sp. CCA e Heterorhabditis sp. JPM3 foi de 28 e $29 \mathrm{JI} / \mathrm{cm}^{2}$, respectivamente. No teste realizado com criptas, ambos os isolados de NEPs foram patogênicos aos insetos.

PALAVRAS-CHAVE: Bioensaios, controle microbiano, Heterorhabditis, Steinernema, praga subterrânea.

\section{ABSTRACT}

PATHOGENICITYOF ENTOMOPATHOGENICNEMATODES AGAINSTTHECOFFEEROOT MEALYBUG DYSMICOCCUS TEXENSIS (TINSLEY) (HEMIPTERA: PSEUDOCOCCIDAE). The coffee root mealybug Dysmicoccus texensis (Tinsley) attacks the roots of this plant and can cause serious damage to crops, and consequent losses in production. The entomopathogenic nematodes (EPNs) are effective agents in controlling pests found in soil, which suggests a likely effectiveness in controlling the coffee root mealybug. Therefore, this study aimed to evaluate the pathogenicity of some strains of EPNs against the coffee root mealybug through selection tests of isolates, determination of the maximum lethal concentration $\left(\mathrm{LC}_{99}\right)$ and the pathogenicity of crypts collected in the field. The isolated Heterorhabditis sp. CCA, Heterorhabditis bacteriophora, Heterorhabditis sp. JPM3.1 and Heterorhabditis sp. JPM3 presented higher virulence, reaching maximum mortality values of $100,94,94$ and $82 \%$ respectively at the highest concentration that was tested (100 infective juveniles IJ/insect). The $\mathrm{LC}_{99}$ determined for Heterorhabditis sp. CCA was estimated at $530 \mathrm{IJ} / \mathrm{plate}$ A similar value was found for Heterorhabditis sp. JPM3, which had the $\mathrm{LC}_{99}$ equal to $560 \mathrm{IJ} / \mathrm{plate}$. Estimating the concentration by area, the value obtained for Heterorhabditis sp. CCA and Heterorhabditis sp. JPM3 was 28 and $29 \mathrm{IJ} / \mathrm{cm}^{2}$, respectively. In the test conducted with crypts, both isolated from EPNs were pathogenic to insects.

KEY WORDS: Bioassays, microbial control, Heterorhabditis, Steinernema, underground pest.

${ }^{2}$ EPAMIG-CTSM/EcoCentro, Lavras, MG, Brasil. 


\section{INTRODUÇÃO}

A cultura do café é uma das mais importantes no Brasil e também de grande significado no mundo, movimentando a economia nacional e internacional. Entretanto, esta cultura sofre perdas consideráveis todos os anos, devido ao grande número de pragas que a atacam (COMPANHIA NACIONAL De AbasteCiMENTO, 2006).

Entre as pragas de maior importância está a cochonilha-da-raiz-do-cafeeiro, Dysmicoccus texensis (Tinsley) (Hemiptera: Pseudococcidae), que se alimenta sugando a seiva das raízes, onde forma colônias que, associadas a fungos do gênero Bornetina, levam à formação de nódulos, criptas ou "pipocas" que impedema absorção deágua e nutrientes pela planta, causando desde o enfraquecimento até a sua morte (Willians; Granara de Willink, 1992; Miller; Polavarapu, 1997; Santa-Cecilia et al., 2002; SantaCecilia et al., 2005).

O controle deste inseto é difícil devido à sua localização no subsolo, o que dificulta a ação de inseticidas e também de inimigos naturais (SANTA-CeCILIA et al., 2005). Os insetos ficam abrigados em volta das raízes da planta, a partir da região do colo, e em altas infestações ficam abrigados nas criptas que se formam nas raízes primárias e secundárias. Além disso, o hábitocríptico dificulta a percepção da sua ocorrência, que muitas vezes é confundida com o ataque de outras pragas (nematóides da raiz) ou ainda coma deficiência de nutrientes, uma vez que os sintomas apresentados pela planta são semelhantes (SouzA et al., 2001; SouzA; Ribeiro, 2003; SANTA-CeCILIA et al., 2005).

$\mathrm{O}$ uso de nematóides entomopatogênicos (Rhabditida: Heterorhabditidae, Steinernematidae) para o controle desse inseto pode ser viável, pois o ambiente ocupado pela cochonilha é bastante semelhante ao exigido pelos nematóides (GrewAL et al., 2001; Lewis et al., 2006).

Algumas espécies de nematóides entomopatogênicos já se mostraram patogênicas a cochonilha-daraiz-do-cafeeiro em testes realizados em laboratório (ANDAló et al., 2004). Entretanto, é necessária a avaliação de um número maior de isolados, já que nos testes realizados até agora a porcentagem máxima de mortalidade foi de $78 \%$. Assim, este trabalho teve como objetivo realizar seleção de isolados denematóides entomopatogênicos para a cochonilha-da-raizdo-cafeeiro, bem como avaliar a concentração letal máxima para os isolados que apresetaram maior virulência sobre o inseto.

\section{MATERIAL E MÉTODOS}

\section{Criação de Dysmicoccus texensis}

A criação de D. texensis foi conduzida no laboratório de Patologia de Insetos da Universidade Federal de Lavras - UFLA, em Lavras, MG, e na Empresa de Pesquisa Agropecuária de Minas Gerais (EPAMIG), Centro Tecnológico do Sul de Minas, localizado também no Campus da UFLA.

A criação permaneceu em condições controladas de temperatura de $27 \pm 1^{\circ} \mathrm{C}$, com umidade relativa de $70 \pm 10 \%$ e fotofase de 12 horas, em câmara do tipo BOD. Como substrato para criação das cochonilhas foram utilizadas batatas com brotações, contendo pelo menos duas fêmeas por brotação e armazenadas em recipientes plásticos, cobertos com filme plástico de PVC perfurado e preso com elástico. A infestação denovas batatas foi feita com auxilio de um pincel fino ou estilete para transferência das cochonilhas, ou colocando batatas novas junto as já infestadas, até que alguns insetos passassem para a nova batata, para que esta pudesse ser removida e colocada em outro recipiente.

Além de batatas, foram usadas abóboras do tipo moranga, variedade "Cabotcha". A manutenção da criação nas abóboras foi semelhante ao procedimento usado nas batatas. Os frutos infestados permaneceram em condições controladas de temperatura de 27 $\pm 1^{\circ} \mathrm{C}$, com umidade relativa de $70 \pm 10 \%$ e fotofase de 12 horas, em câmara do tipo BOD. Para infestação de novas abóboras, foram colocados, sobre ela, pedaços de uma abóbora já infestada, esperando pela passagem das ninfas e adultos da cochonilha.

\section{Obtenção dos isolados de nematóides entomopatogênicos}

Os isolados foram obtidos no Banco de Entomopatógenos do Laboratório de Patologia de Insetos da UFLA (Tabela 1), onde permaneceram armazenados em frascos Erlenmeyer na forma de suspensão aquosa, sob condições controladas a $16^{\circ} \pm$ $1^{\circ} \mathrm{C}$, com umidade relativa de $70 \pm 10 \%$, na concentração de até 500 juvenis infectantes (JI)/mL.

Quando necessária, a multiplicação foi feita em lagartas de último instar da traça dos favos, Galleria mellonella. Para multiplicação dos isolados foi utilizada a metodologia descrita por POINAR JUNIOR (1979), ondea infecção delarvas deúltimoínstar de G. mellonella com JI é realizada por meio do sistema de infecção tópica. Após a infecção, as larvas foram incubadas a 25 $\pm 2^{\circ} \mathrm{C}$ e transferidas para câmara seca. Armadilhas de White (WHITE, 1927) foram usadas para obtenção dos nematóides sobas mesmas condições. Para montagem dosexperimentos, os JIemergidosforamarmazenados sob temperatura de $16^{\circ} \pm 1^{\circ} \mathrm{C}$ por, no máximocinco dias.

\section{Seleção dos isolados de nematóides entomopatogênicos}


Tabela 1 - Linhagens e origem dos nematóides entomopatogênicos utilizados no teste de seleção de isolados.

\begin{tabular}{lll}
\hline Linhagem & Sigla & Local \\
\hline Steinernema (= anomali) arenarium & $\mathrm{Sa}$ & Voronezh / Rússia \\
Steinernema carpocapsae All & $\mathrm{Sc}$ & Carolina do Norte / USA \\
Steinernema riobravis 355 & $\mathrm{Sr}$ & Texas / USA \\
Steinenema glaseri NA & $\mathrm{Sg}$ & Flórida / USA \\
Heterorhabditis bacteriophora & $\mathrm{Hb}$ & New Jersey / USA \\
Heterorhabditis bacteriophora HP88 & $\mathrm{HP88}$ & New Jersey / USA \\
Heterorhabditis sp. (CCA) & $\mathrm{CCA}$ & Araras / SP / Brasil \\
Heterorhabditis sp. (JPM4) & $\mathrm{JPM} 4$ & Lavras / MG / Brasil \\
Heterorhabditis sp. (JPM3.1) & $\mathrm{JPM} 3.1$ & Lavras / MG / Brasil \\
Heterorhabditis sp. (JPM3) & $\mathrm{JPM}$ & Lavras / MG / Brasil \\
Heterorhabditis sp. (PI) & $\mathrm{PI}$ & Teresina / PI / Brasil \\
\hline
\end{tabular}

Os isolados testados foram quatro do gênero Steinernema (S. glaseri, S. anomali, S. carpocapsae e $S$. riobravis) e sete do gênero Heterorhabditis ( $H$. bacteriophora, H. bacteriophora HP88, Heterorhabditis habditis sp. CCA, Heterorhabditis sp. JPM3, Heterorhabditis sp. JPM3.1, Heterorhabditis sp. JPM4 e Heterorhabditis sp. PI).

Cada isolado foi testado em três concentrações (25, 50 e $100 \mathrm{JI} /$ inseto), resultando num delineamento experimental fatorial $3 \times 11$. Cada tratamento teve cinco repetições, sendo cada uma delas composta por dez cochonilhas fêmeas adultas. Foram utilizadas placas de Petri ( $5 \mathrm{~cm}$ de diâmetro) com $20 \mathrm{~g}$ de areia esterilizada e broto de batata, sobre o qual foram dispostos os dez insetos. Cada placa recebeu $1 \mathrm{~mL}$ de suspensão. Após a inoculação, os brotos foram recobertos com a areia e as placas acondicionadas em caixas plásticas contendo espuma embebida emágua destilada para manutenção da umidade.

O experimento foi mantido em B.O.D. a $25^{\circ} \mathrm{C}, 70 \pm$ $10 \%$ de umidade e fotofase de 12 horas. Além dos tratamentos que receberam os nematóides, foi também montado um tratamento adicional que recebeu apenas água destilada (testemunha).

A avaliação foi realizada após cinco dias, armazenando as cochonilhas mortas em câmara seca, para posterior confirmação da morte causada pelos nematóides entomopatogênicos através da observação da sintomatologia (PoINARJunIOR, 1990; BOEMARE, 2002).

Os dados de mortalidade foram submetidos à análise de variância e ao teste de média Scott-Knott (P $<0,05)$ para comparação entre as médias.

\section{Concentração Letal Máxima $\left(\mathrm{CL}_{99}\right)$}

Após a seleção dos isolados, foram escolhidos os dois (Heterorhabditis sp. CCA e Heterorhabditis sp. JPM3) que se mostraram mais virulentos a cochonilha-da-raiz-do-cafeeiro para utilizá-los nos testes subseqüentes. Para determinação da concentração letal máxima $\left(\mathrm{CL}_{99}\right)$ foram avaliadas 10 concentrações: 50, 100, 250, 500, 750,1.000,1.250,1.500, 1.750 e $2.000 \mathrm{JI} /$ placa aplicados em $2 \mathrm{~mL}$ de água destilada. Cada tratamento foi repetido quatro vezes, e cada parcela consistiu de uma placa de Petri ( $5 \mathrm{~cm}$ de diâmetro) contendo areia e um broto de batata, sobre o qual foram dispostas 10 fêmeas adultas da cochonilha. A testemunha recebeu apenas água destilada. Os dados foram submetidos à análise de regressão pelo programa Sigma-Plot (2003). Para estimação da $\mathrm{CL}_{99}$ foi utilizada a equação de regressão derivada, estimando-se o valor desejado no intervalo avaliado.

\section{Eficiência dos isolados sobre criptas}

Para avaliação da patogenicidade sobre criptas, foram usados os dois isolados que apresentaram melhores resultados no teste de seleção de isolados (CCA e JPM3). As criptas foram coletadas no campo, cortadas em pedaços de $1,5 \mathrm{~cm}^{2}$ e colocadas em placas de Petri ( $5 \mathrm{~cm}$ de diâmetro) contendo $20 \mathrm{~g}$ de areia. A concentração usada foi de $500 \mathrm{JI} /$ inseto. Para cada tratamento foram feitas 5 repetições, e a avaliação foi feita após 5 dias, sendo os insetos mortos transferidos para câmara seca visando a confirmação da mortalidade através da sintomatologia (PoinAR Junior, 1990; Boemare, 2002). A testemunha recebeu apenas água destilada.

Os dados foram submetidos à análise de variância e ao teste de média Scott-Knott $(\mathrm{P}<0,05)$ para comparação entre as médias.

\section{RESULTADOS E DISCUSSÃO}

\section{Seleção dos isolados de nematóides entomopatogênicos}


Verificou-se que todos os isolados testados foram patogênicos à cochonilha-da-raiz-do-cafeeiro nas três concentrações testadas. Os isolados pertencentes ao gênero Steinernema foram menos virulentos do que os do gênero Heterorhabditis (Tabela 2).

Já para heterorhabditídeos, os isolados Heterorhabditis sp. CCA, H. bacteriophora, Heterorhabditis sp. JPM3.1 e Heterorhabditis sp. JPM3, foram os que apresentaram maior virulência alcançando valores máximos de mortalidade de 100, 94, 94 e $82 \%$ respectivamente, na maior concentração testada (100 JI/inseto).

Na menor concentração testada (25 JI/inseto), o isolado JPM3 foi o que causou maior mortalidade (84\%) dos insetos. A concentração com menor variação entre os isolados testados foi a de $50 \mathrm{JI} /$ inseto, sendo nítida a diferença de virulência entre os isolados de heterorhabditídios e esteinernematídios.

Esses resultados foram semelhantes aos obtidos porStUART etal. (1997) que, avaliando a suscetibilidade de Dysmicoccus vacinii Miller \& Polavarapu (Hemiptera: Pseudococcidae) a diferentes isolados de nematóides entomopatogênicos, observaram a maior suscetibilidade deste inseto às espécies pertencentes ao gênero Heterorhabditis, com valores de até $90 \%$ de mortalidade.

Diferentemente, ANDALó et al. (2004), realizando trabalho de seleção de isolados de nematóides entomopatogênicos para $D$. texensis, porém, usando uma metodologia diferenciada, observaram que $S$. carpocapsae foi o que se mostrou mais virulento, causando até $78 \%$ de mortalidade. Estes dados diferem dos obtidos, onde $S$. carpocapsae causou apenas $20 \%$ de mortalidade, mesmo nas maiores concentrações testadas.

Os resultados demonstram que houve grande variabilidade na suscetibilidade de $D$. texensis aos diferentes isolados testados, com resultados que variam de apenas $2 \%$ de mortalidade (S. riobravis na concentração de 25JI/placa) a até100\% (Heterorhabditis sp. CCA na concentração de $100 \mathrm{JI} /$ placa). Essas diferenças reforçam a necessidade de testes de seleção com isolados de nematóides entomopatogênicos, pois as características e adaptações que cada isolado possui em relação ao ambiente e ao hospedeiro podem variar enormemente (GAUGLER et al., 1997).

Vários fatores justificam as diferenças de virulência entre isolados da mesma espécie. Isolados de diferentes locais podem estar adaptados a diferentes condições climáticas, ou mesmo ter desenvolvido especificidade a hospedeiros locais, o que é evidenciado no experimento, uma vez que os isolados autóctones (Heterorhabditis sp. JPM3, Heterorhabditis sp. JPM4 e Heterorhabditis sp. CCA) demonstraram maior virulência contra o inseto quando comparado com $H$. bacteriophora, que foi menos virulento.

A maior suscetibilidade aos heterorhabditídios do que aos esteinernematídios pode ser, em parte, explicada pelo menor tamanho que estes possuem. Segundo STUART et al. (1997), os esteinernematídios podem ter dificuldades de penetrar pelas aberturas naturais de insetos menores, como é o caso da cochonilha-da-raiz-do-cafeeiro. Entretanto, uma serie de outros fatores podem influenciar como, por exemplo, especificidade entre patógeno e hospedeiro.

Tabela 2 - Porcentagem de mortalidade de fêmeas adultas da cochonilha-da-raiz-do-cafeeiro Dysmicoccus texensis causada por diferentes isolados de nematóides entomopatogênicos em três diferentes concentrações.

\begin{tabular}{lccc}
\hline Isolados & \multicolumn{3}{c}{ Concentração (JI/ Inseto) } \\
\cline { 2 - 4 } & 25 & 50 & 100 \\
\hline Testemunha & $4,0 \pm 5,7 \mathrm{E}^{1}$ & $4,0 \pm 5,7 \mathrm{C}$ & $4,0 \pm 5,7 \mathrm{~F}$ \\
Steinernema carpocapsae & $20,0 \pm 7,47 \mathrm{D}$ & $18,0 \pm 0,13 \mathrm{~B}$ & $24,0 \pm 13,33 \mathrm{E}$ \\
Steinernema. anomali & $30,0 \pm 8,33 \mathrm{D}$ & $28,0 \pm 3,33 \mathrm{~B}$ & $36,0 \pm 7,50 \mathrm{D}$ \\
Steinernema riobravis & $2,0 \pm 3,20 \mathrm{E}$ & $20,0 \pm 8,00 \mathrm{~B}$ & $22,0 \pm 10,40 \mathrm{E}$ \\
Steinernema glaseri & $12,0 \pm 6,67 \mathrm{E}$ & $24,0 \pm 5,00 \mathrm{~B}$ & $34,0 \pm 9,17 \mathrm{D}$ \\
Heter bacteriophora & $42,0 \pm 13,60 \mathrm{C}$ & $90,0 \pm 4,00 \mathrm{~A}$ & $94,0 \pm 7,20 \mathrm{~A}$ \\
Heterorhabditis HP88 & $30,0 \pm 8,00 \mathrm{D}$ & $20,0 \pm 4,00 \mathrm{~B}$ & $40,0 \pm 16,00 \mathrm{D}$ \\
Heterorhabditis CCA & $72,0 \pm 11,67 \mathrm{~B}$ & $98,0 \pm 3,33 \mathrm{~A}$ & $100,0 \pm 0,00 \mathrm{~A}$ \\
Heterorhabditis JPM3 & $84,0 \pm 11,91 \mathrm{~A}$ & $84,0 \pm 7,66 \mathrm{~A}$ & $82,0 \pm 15,32 \mathrm{~B}$ \\
Heterorhabditis JPM3.1 & $48,0 \pm 6,81 \mathrm{C}$ & $90,0 \pm 8,61 \mathrm{~A}$ & $94,0 \pm 7,66 \mathrm{~A}$ \\
Heterorhabditis JPM4 & $66,0 \pm 9,36 \mathrm{~B}$ & $80,0 \pm 8,51 \mathrm{~A}$ & $62,0 \pm 6,81 \mathrm{C}$ \\
Heterorhabditis PI & $62,0 \pm 6,81 \mathrm{~B}$ & $80,0 \pm 8,51 \mathrm{~A}$ & $82,0 \pm 14,47 \mathrm{~B}$ \\
\hline
\end{tabular}

$\mathrm{CV}=22,64$

${ }^{1}$ Médias seguidas por letras distintas nas colunas diferem entre si pelo teste Scott-Knott $(\mathrm{P}<0,05)$. 


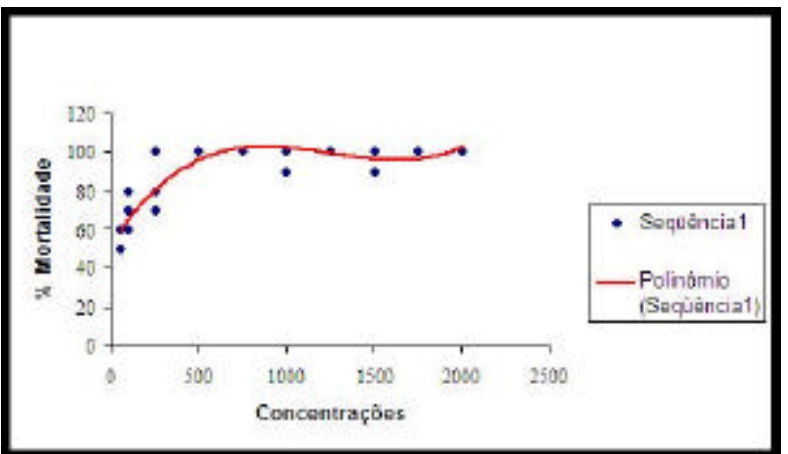

Fig. 1 - Curva de regressão para o isolado Heterorhabditis sp. CCA considerando o número de juvenis infectantes/ placa necessário para causar $99 \%$ de mortalidade $\left(\mathrm{CL}_{99}\right)$. $\breve{y}=3-08 x^{3}-0,0001 x^{2}+0,1396 x+52,58$.

A especificidade que cada isolado possui sobre determinado hospedeiro está diretamente ligada a sua eficiência em alcançar o hospedeiro, penetrar nele ecausar doença, além dedriblar o sistema imunológico do inseto, para que este não seja capaz de combatê-lo. Essa especificidade é um processo bem complexo, mas cuja compreensão pode esclarecer o porquê de tanta variabilidade na eficiência de diferentes espécies ou mesmo isolados sobre determinados hospedeiros (Lewis et al., 2006).

Assim, a co-evolução entre as espécies pode proporcionar odesenvolvimento demecanismos dereconhecimento do hospedeiro pelos nematóides, como percepção química ou desenvolvimento de estruturas físicas especificas que auxiliem no processo de infecção. Quanto aos estímulos químicos, os nematóides entomopatogênicos podem reconhecer sinais emitidos pelo próprio hospedeiro ou por plantas por eleatacadas (emissão de $\mathrm{CO}_{2}$, por exemplo) e deslocar-se em direção dos estímulos recebidos (van Tol et al., 2001).

A diferença na susceptibilidade de $D$. texensis as diferentes espécies e isolados avaliados reforçou a necessidade da realização de testes de seleção, demonstrando que diferentes espécies apresentam maior ou menor especificidade em atacar o inseto, e que isolados nativos podem ter maior virulência sobre o inseto do que espécies exóticas.

\section{Estimativa da Concentração Letal Máxima $\left(\mathrm{CL}_{99}\right)$}

Para Heterorhabditis sp. CCA a $\mathrm{CL}_{99}$ foi estimada em 530 (JI)/ placa (Fig. 1). Valor semelhante foi encontrado para Heterorhabditis sp. JPM3 que teve a $\mathrm{CL}_{99}$ igual a $560 \mathrm{JI} /$ placa (Fig. 2). Em trabalho realizado por STUART et al. (1997), para avaliação da sucetibilidade de D. vaccinii, os autores alcançaram valor máximo de mortalidade de $83 \%$ aplicando $500 \mathrm{JI} /$ placa.

Estimando a concentração por área, o valor obtido para esses nematóides foi de 28 e $29 \mathrm{JIs} / \mathrm{cm}^{2}$, respec-

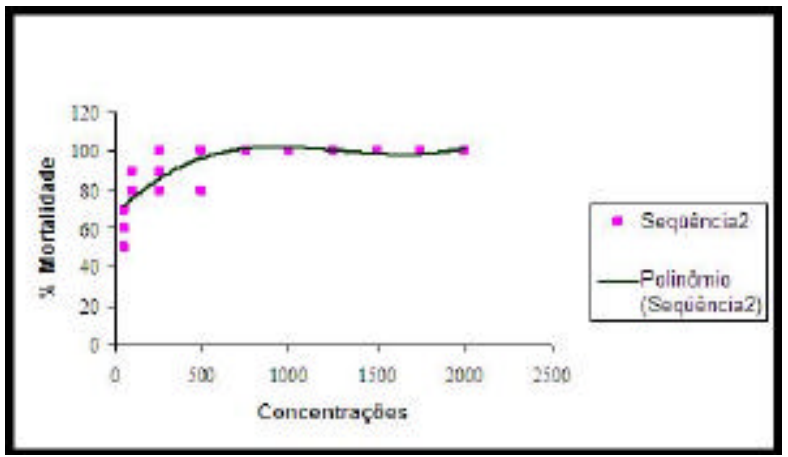

Fig. 2 - Curva de regressão para o isolado Heterorhabditis sp. JPM3 considerando o número de juvenis infectantes / placa necessário para causar $99 \%$ de mortalidade $\left(\mathrm{CL}_{99}\right)$. $\breve{y}=2-08 x^{3}-8-05 x^{2}+0,0939 x+66,69$.

tivamente. Quandoa concentraçãoéextrapolada para aplicações a campo o valor é de 2,8 x $10^{9} \mathrm{JI} /$ ha. Este valor é inferior ao encontrado em outros trabalhos, onde recomendam-se aplicações de $1 \times 10^{10} \mathrm{JI} / \mathrm{ha}$ (Thurston et al., 1994; EBssa et al., 2004; Siegel et al., 2004).

Por outro lado, a concentração determinada em laboratório pode não ser adequada em condições de campo onde o número de fatores não controlados é maior. Em condições de laboratório, o inseto fica altamente propenso ao ataque do nematóide, enquanto que em condições de campo o nematóide fica exposto a uma série de intempéries, como variação da temperatura e da umidade, além de ter que buscar pelo hospedeiro.

\section{Avaliação da virulência contra insetos alojados nas criptas}

Os dois isolados testados foram virulentos contra a cochonilha-da-raiz-do-cafeeiro abrigada em criptas (Tabela 3). O isolado JPM3 causou até 74\% de mortalidade, enquanto que CCA matou $93 \%$ dos insetos, sendo, portanto, mais virulento.

Tabela3-Porcentagem demortalidadedeDysmicoccustexensis em criptas inoculadas com dois isolados de nematóides entomopatogênicos na concentração de $500 \mathrm{JI} /$ placa.

\begin{tabular}{lc}
\hline Isolado & Mortalidade (\%) \\
\hline Testemunha & $8,2 \pm 0,04 \mathrm{C}^{1}$ \\
Heterorhabditis sp. JPM3 & $74,2 \pm 0,08 \mathrm{~B}$ \\
Heterorhabditis sp. CCA & $93,7 \pm 0,10 \mathrm{~A}$ \\
\hline
\end{tabular}

$\mathrm{CV}=19,69$

${ }^{1}$ Medias seguidas de letras distintas diferem entre si pelo teste Scott-Knott $(\mathrm{P}<0,05)$. 
A alta susceptibilidade que os insetos apresentaram aos nematóides, mesmo quando abrigados nas criptas, é um bom indício da possibilidade de uso destes agentes no controle do inseto em condições de campo, apesar de que, neste experimento, as criptas foram cortadas, facilitando o acesso dos JI.

Desta maneira, este trabalho demonstrou que a realização de testes de seleção de isoladoséimportante, haja vista a grande variação que houve quanto a sua virulência sobre $D$. texensis, bem como a realização de teste complementares, para melhor caracterização dos isolados.

\section{AGRADECIMENTOS}

ÀCAPES pela concessão de bolsa, permitindo que este trabalho fosse realizado. À EPAMIG, empresa financiadora do projeto.

\section{REFERÊNCIAS}

ANDALÓ, V.; MOINO JUNIOR, A.; SANTA-CECILIA, L.V.C.; SOUZA, G.C. Seleção de isolados de fungos e nematóides entomopatogênicos pra a cochonilha-daraiz-do-cafeeiro Dysmicoccus texensis (Tinsley). Arquivos do Instituto Biológico, São Paulo, v.71, n.2, p.181-187, 2004.

BOEMARE, N. Biology, Taxonomy and Systematics of Photorhabdus and Xenorhabdus. In: GAUGLER, R. (Ed.). Entomopathogenic nematology. Wallingford, UK: CABI Publishing, 2002. Chap.2, p.35-56.

\section{COMPANHIA NACIONAL DE ABASTECIMENTO.}

Brasil. Indicadores da agropecuária. Brasília. Disponível em: <http://www.receita.rj.gov.br/legislacao/ indice/c/companhia_nacional_de_abastecimento. shtml >. Acesso em: 11 mar. 2006.

EBSSA, L.; BORGEMEISTER, C.; POEHLING, H-M. Effectiveness if different species/strains of entomopathogenic nematodes for control of wester flower thrips (Frankliniella occidentalis) at various concentrations, host densities, and temperatures. Biological Control, v.29, p.145-154, 2004.

GAUGLER, R.; LEWIS, E.; STUART, R.J. Ecology in the service of biological control: the case of entomopathogenic nematodes. Oecologia, v.109, p.483$489,1997$.

GREWAL P.G.; NARDO, E.A.B. DE; AGUILLERA, M. Entomopathogenic nematodes: Potential for exploration and use in south america. Neotropical Entomology, v.30, p.191-205, 2001.
LEWIS, E.D.; CAMPBELL, J.; GRIFFIN, C.; KAYA, H.; PETERS, A. Behavioral ecology of entomopathogenic nematodes. Biological Control, v.38, n.1, p.66-79, 2006.

MILLER, R.D.; POLAVARAPU, S.A. A new species of mealbug in the genus Dysmicoccus (Hemiptera: Coccoidea: Pseudococcidae) of importance in highbush blueberries (Vaccinium corymbosum, Ericaceae) in the Eastern United States. Proceedings of the Entomological Society of Washington, v.99, p.440-460, 1997.

POINAR JUNIOR, G.O. Nematodes for biological control of insects. Boca Raton: CRC Press, 1979.

POINAR JUNIOR, G.O. Taxonomy and biology of Steinernematidae and Heterorhabditidae. In.: GAUGLER, R.; KAIA, H.K. (Ed.). Entomopathogenic nematodes in biological control. Boca Raton: CRC Press, 1990. p.23-66.

SANTA-CECILIA, L.V.C.; REIS, P.R.; SOUZA, J.C. Sobre a nomenclatura das espécies de cochonilhasfarinhentas do cafeeiro nos estados de Minas Gerais e Espírito Santo. Neotropical Entomology, v.31, p.333-334, 2002.

SANTA-CECILIA, L.V.C.; SOUZA, B.; PRADO, E.; SOUZA, J.C.; FORNAZIER, M.J. Cochonilhas-farinhentas em cafeeiros: reconhecimento e controle. Lavras: EPAMIG, 2005. (Circular Técnica, $\mathrm{n}^{\circ}$ 189).

SIEGEL, J.; LACEY, L.A.; FRITTS JUNIOR, R.; HIGBEE, B.S.; NOBLE, P. Use of steinernematid nematodes for post harvest control of navel orangeworm (Lepidoptera: Pyralidae, Amyelois transitella) in fallen pistachios. Biological Control, v.30, p.410-417, 2004.

SOUZA, J.C.; REIS, P.R.; SANTA-CECILIA, L.C.V.; DAUM, S.; SOUZAM M. de A. Cochonilha-da-raiz do cafeeiro: aspectos biológicos, dano e controle. Lavras: EPAMIG, 2001. 4p. (Circular Técnica).

SOUZA, J.C.; RIBEIRO, J.A. Cochonilha-da-raiz: cafeicultor, conheça e saiba como controlar esta praga com inseticidas neonicotinóides. Lavras: EPAMIG, 2003. 3p.(Circular Técnica, $\mathrm{n}^{\mathrm{o}} 162$ ).

STUART, R.J.; POLAVARAPU, S.; LEWIS, E.E.; GAUGLER, R. Differential susceptibility of Dysmicoccus vacinni (Homoptera: Pseudococcidae) to entomopathogenic nematodes (Rhabdita: Heterorhabditidae and Steinernematidae). Journal of Economic Entomology, v.90, p.925-932, 1997.

THURSTON, G.S.; KAYA, H.K.; GAUGLER, R. Characterizing the enhanced susceptibility of milky disease-infected scarabaeid grubs to entomopathogenic nematodes. Biological Control, v.4, p.67-73, 1994 .

van TOL, R.W.H.M.; van der SOMMEN, A.T.C.M.; BOFF, I.C.; van BEZOOIJEN, J.; SABELIS, M.W.; SMITS, 
P.H. Plants protect their roots by alerting the enemies of grubs. Ecologic Letter, v.4, p.292-294, 2001.

WILLIANS, D.J.; GRANARA de WILLINK, M.C. Mealybugs of central and south América. Wallingford: CABI, 1992. 629p.
WHITE, G.F. A method for obtaining infective nematode larvae from cultures. Science, v.66, p.302303, 1927.

Recebido em 19/1/07

Aceito em 20/11/08 